\title{
Obstructive uropathy secondary to a ureteral paraperitoneal inguinoscrotal hernia in a patient with a pelvic ectopic solitary kidney and malrotation: a case report and review of the literature
}

Juan Camilo Álvarez Restrepo ${ }^{*}$, Susan Julieth Moreno Diaz², Sthepani Gómez Castro ${ }^{3}$, Carlos Andres Riveros ${ }^{4}$, Daniel Salazar Radi ${ }^{5}$ and Jhonny Bustos Calvo ${ }^{6}$

\begin{abstract}
Background: Ureteral inguinoscrotal hernias (UISH) are rare, associated in some cases with congenital abnormalities of the urinary tract. Their presentation is commonly asymptomatic, and diagnosis is mostly incidental.

Case presentation: A 65-year-old male patient presented to the emergency room with neurologic symptoms and an altered renal function. Further investigation revealed a ureteral paraperitoneal inguinoscrotal hernia with a pelvic ectopic solitary kidney and malrotation. Three days after hernia repair and placement of a ureteral JJ stent, the creatinine levels stabilized, and the patient was discharged.

Conclusion: This is the first reported case of a ureteral paraperitoneal inguinoscrotal hernia in a patient with a pelvic ectopic solitary kidney and malrotation. Currently, it is also the only case reported that has been managed by laparoscopy.
\end{abstract}

Keywords: Inguinal hernia, Ureter, Scrotum, Congenital abnormalities, Case report

\section{Background}

Ureteral inguinoscrotal hernias (UISH) are rare, with less than 140 cases reported in the literature [1]. They are more common in obese men and kidney transplant recipients between the fifth and sixth decades of life; right-sided involvement is predominant [2-5]. The paraperitoneal and extraperitoneal subtypes have been described, the latter related to urinary tract abnormalities. They are generally asymptomatic, and the diagnosis is often incidental on imaging or intraoperative due

\footnotetext{
*Correspondence: juancalvarezr92@hotmail.com

${ }^{1}$ Department of Urology, School of Medicine, Universidad Nacional de

Colombia, Carrera 30 No. 45-03, Edificio 471, Bogotá, Colombia

Full list of author information is available at the end of the article
}

to ureteral injury [1]. There are no treatment guidelines; the management seeks to correct the hernia defect and obstructive uropathy, in some cases conservative management and follow-up can be performed [6-8]. We report the first case of a ureteral paraperitoneal inguinoscrotal hernia in a patient with a pelvic ectopic solitary kidney and malrotation.

\section{Case presentation}

A 65-year-old male patient presented to the emergency department with neurologic symptoms and was admitted for an acute pure motor lacunar stroke, National Institutes of Health Stroke Scale (NIHSS) 1, Alberta Stroke Program Early CT Score (ASPECTS) 10/10. The patient lacked insight regarding his medical condition, and there 
were no family members present. However, known past medical history included hypertension and kyphoscoliosis. During the initial evaluation of the patient, the admitting service evidenced a creatinine level of $4.18 \mathrm{mg} / \mathrm{dL}$ with an estimated glomerular filtration rate (eGFR) of $14 \mathrm{ml} / \mathrm{min} / 1.73 \mathrm{~m}^{2}$ (Chronic Kidney Disease Epidemiology Collaboration [CKD-EPI]), for which they requested a renal and urinary tract ultrasound (US), documenting a solitary heterotopic pelvic ectopic solitary kidney and grade 4 hydronephrosis. Due to these findings, the admitting service requested a computed tomography urogram (CTU) and a urology consultation.

During the physical examination by the urology service, the patient had normal vital signs. A focused urologic exam revealed a urethral catheter with clear urine drainage, and an increase in the right hemiscrotal volume compared to the left one, with reducible content along with a positive transillumination test compatible with an indirect inguinal hernia (Fig. 1). It was striking to observe how reducing the content of the hernia increased urine drainage through the urethral catheter, quantifying approximately $150 \mathrm{~mL}$ in five minutes. Urinalysis results were unremarkable.

Images of the patient's CTU were evaluated, showing the absence of the left kidney, with a solitary right pelvic kidney, and malrotation with marked dilation of the collecting system and right ureter throughout its entire trajectory. In addition, there was a right inguinoscrotal hernial sac with the right ureter bent inside along with omentum (Figs. 2 and 3). Due to the altered renal

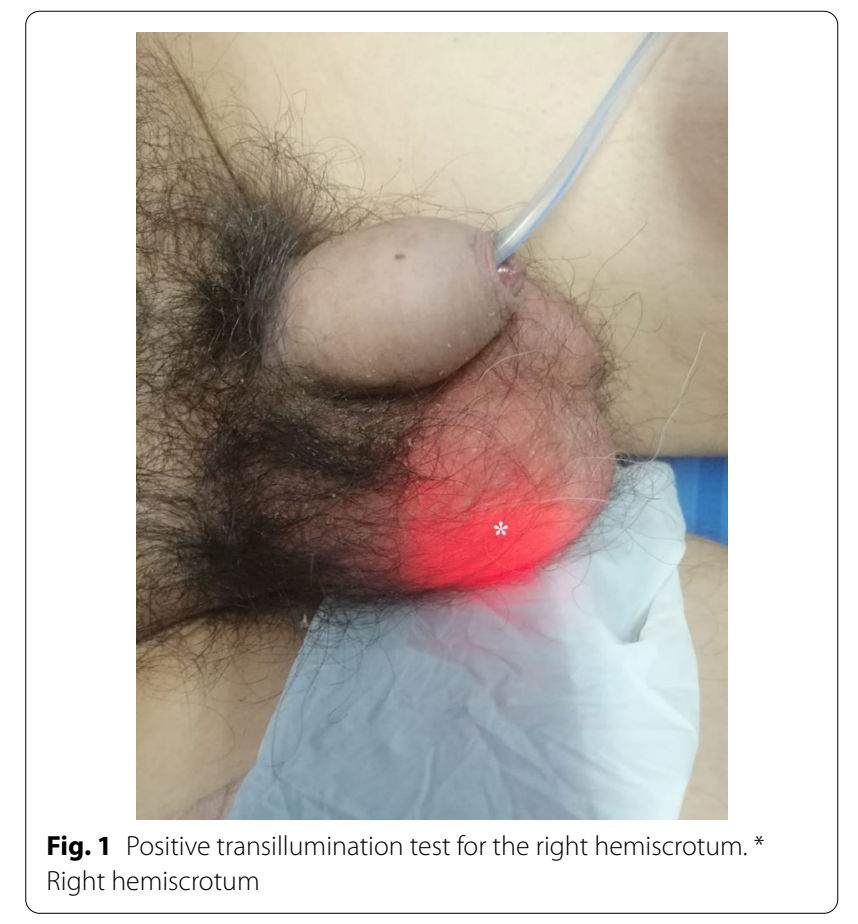

Fig. 1 Positive transillumination test for the right hemiscrotum. * Right hemiscrotum

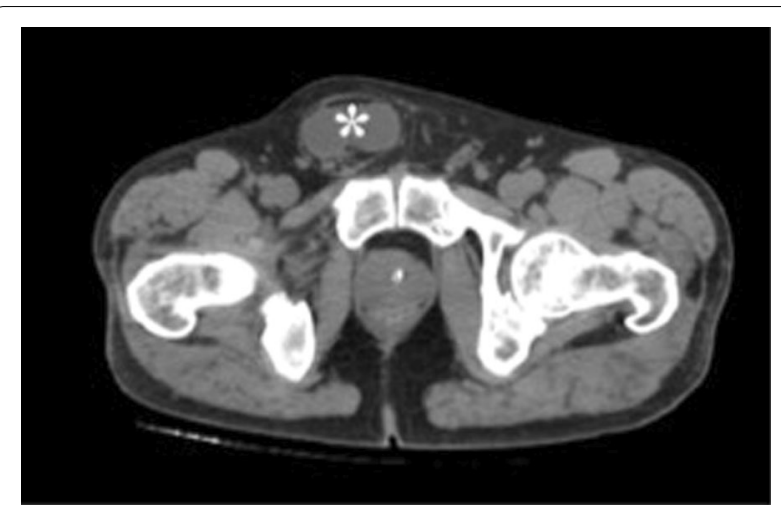

Fig. 2 Axial section of the CTU, showing herniation of the ureter through the right inguinal canal. * Right inguinal canal

function and findings on imaging, the patient was programmed for surgery.

On account of the presence of a right UISH, surgical cooperation between the general surgery and urology departments was arranged. The passage of a ureteral JJ stent was initially performed without complications, with findings on ureteroscopy of a right ureter with a dilated intraluminal tract with multiple kinks. During the same surgical time, intraperitoneal laparoscopic herniorrhaphy with synthetic mesh was performed. Intraoperatively, a right indirect inguinal hernia of $3 \mathrm{~cm}$ in diameter was found containing the right ureter, identified by the presence of the JJ stent (Fig. 4). These

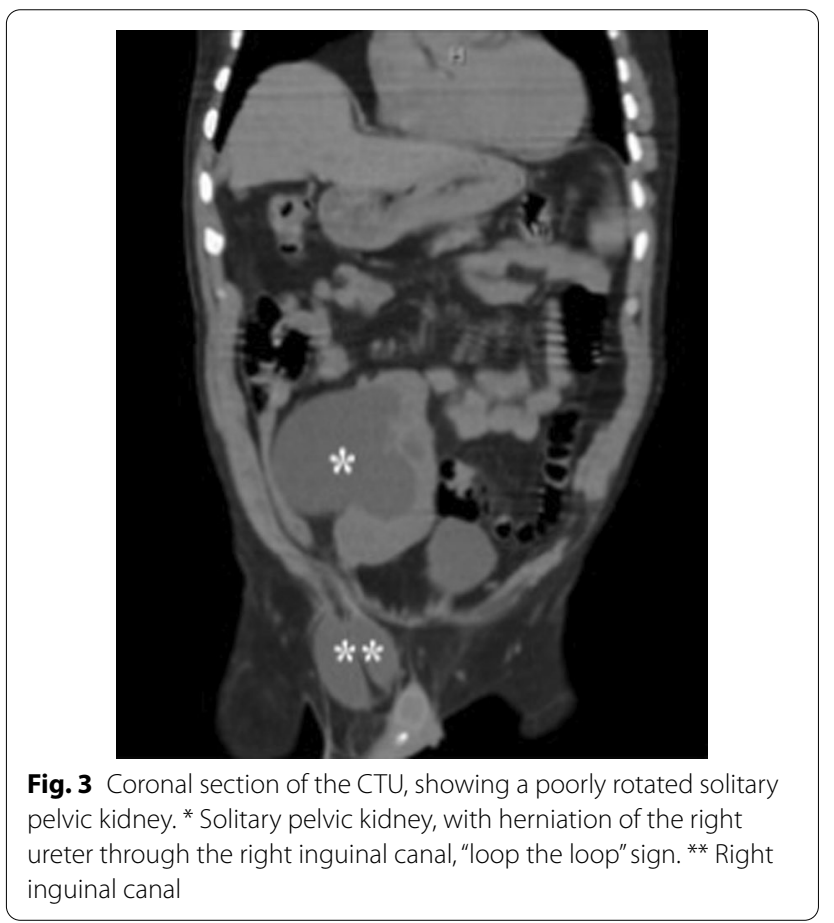




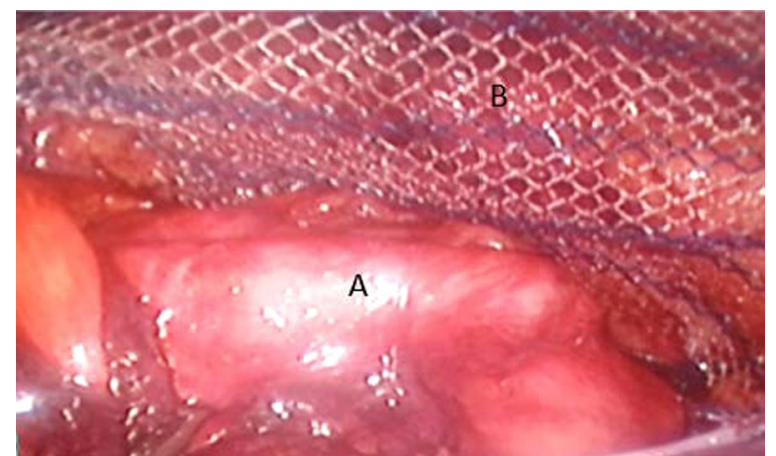

Fig. 4 The lower part shows a ureter with a JJ stent in place $(\mathbf{A})$. The upper part shows a polypropylene mesh over the hernial defect in its proper position (B)

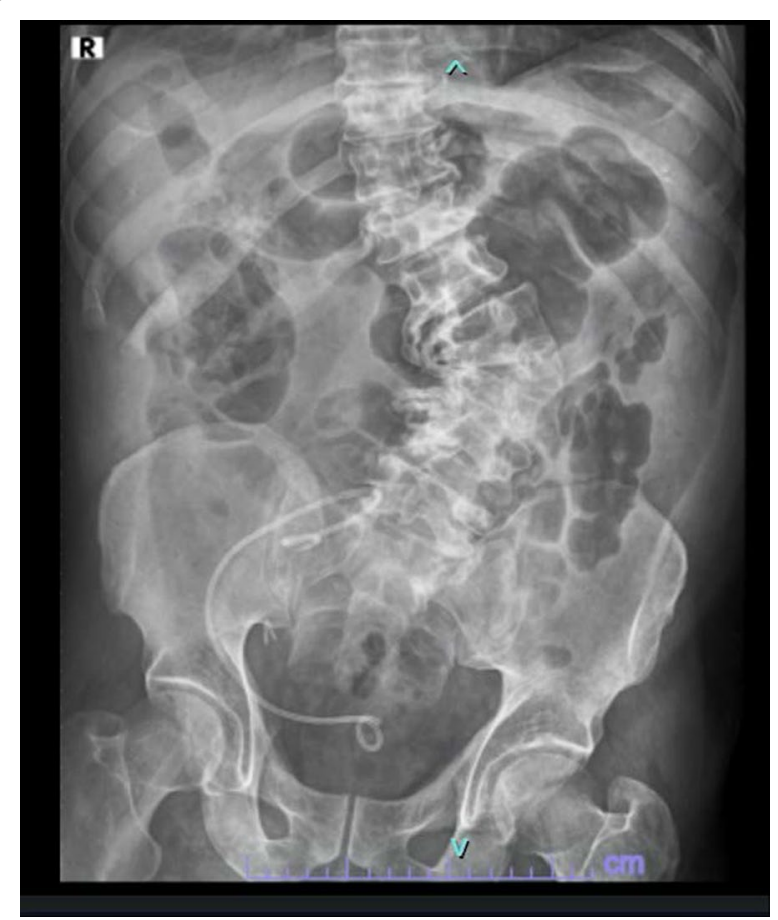

Fig. 5 Plain abdominal X-ray: J catheter in normal position

findings were consistent with a ureteral paraperitoneal inguinoscrotal hernia.

While the patient continued under clinical surveillance, laboratory exams showed stabilization of creatinine levels with a value of $3.05 \mathrm{mg} / \mathrm{dL}$ on postoperative day 3 (eGFR $20 \mathrm{ml} / \mathrm{min} / 1.73 \mathrm{~m}^{2}$ using CKD-EPI). Plain abdominal radiography revealed an adequately positioned JJ stent (Fig. 5). The patient was discharged after three uneventful days with an indication to return one month later for JJ stent removal, but unfortunately, the patient was lost to follow-up.

\section{Discussion}

Inguinal hernias are one of the most frequently found surgical pathologies in clinical practice; more than 20 million people worldwide undergo repair annually [9]. The lifetime risk of developing an inguinal hernia is $27 \%$ in men and 3\% in women [10].

Indications for management include pain relief or as a surgical emergency, to mitigate the appearance of secondary complications. Surgical treatment is successful in most cases, with a reoperation rate of $10-15 \%$ and chronic pain in approximately $10-12 \%$ of patients [9].

Unusual presentations of inguinal hernias have been described, which are reported according to their content; examples include Amyand's hernia (cecal appendix), Littre's hernia (Meckel's diverticulum), and Richter's hernia (wall of the small intestine) $[1,10]$.

Among the unusual presentations is the UISH, called a sliding hernia because of its retroperitoneal content. It was first described in 1880 and since then only approximately 140 cases have been reported globally [1].

Ureteral hernias are a rare pathology of the urinary system; their most common presentation is inguinoscrotal. They are more common in men between the fifth and sixth decades of life, with a higher number of cases of femoral ureteral hernias being described in women [4, 5]. They predominate on the right side since the sigmoid mesocolon on the left side maintains the ipsilateral ureter retroperitoneally. Eighty percent are indirect hernias and only $20 \%$ are direct $[1,11]$.

Two subtypes of UISH are known, paraperitoneal and extraperitoneal. The paraperitoneal subtype is the most common and presents adherence to the posterior peritoneum with large hernial defects, described in up to $80 \%$ of cases $[3,11]$. On the other hand, the extraperitoneal subtype represents the remaining $20 \%$ of cases. It is considered congenital due to anomalies in the development of the Wolffian ducts; they do not have a peritoneal sac but have retroperitoneal fat content $[6,13]$. The latter has a relationship of up to $46 \%$ with urinary tract abnormalities such as crossed renal ectopia, nephroptosis, and horseshoe kidney $[2,13,14]$.

Ureteral inguinoscrotal hernias are generally asymptomatic; the most common finding is a scrotal mass and $50 \%$ present obstructive symptoms of the upper urinary tract. The diagnosis is made in a lower percentage during a preoperative evaluation or as an incidental intraoperative finding due to ureteral injury $[1,11]$.

Despite being a rare pathology, it is recommended that in the presence of impaired kidney function or urinary tract infection (particularly in men), a renal and urinary 
tract US is performed to determine the existence of hydronephrosis. Figure 6 presents a diagnostic algorithm adapted from Yahya et al. [1]. Classical imaging findings include the "loop the loop" sign on pyelogram and the "fish hook" sign on abdominal computed tomography (CT) $[1,11,14]$.

Some recognized risk factors for ureteral inguinoscrotal hernias are male sex, obesity, collagen synthesis defects, and a history of kidney transplantation [2,3]. An important series of cases reported an anterior displacement of the ureter from the psoas muscle by at least $1 \mathrm{~cm}$ at the level of $L 4$ in all patients $[4,15]$.

The management of these patients seeks to correct the hernial defect and the associated obstructive uropathy. The surgical technique describes the placement of a JJ stent prior to mesh herniorrhaphy to facilitate intraoperative identification of the ureter and renal decompression in case of obstructive uropathy [6, 7]. Likewise, the aim is to rearrange the ureter in its retroperitoneal trajectory and in cases of redundant and tortuous ureters, shortening with ureteroureterostomy or a ureteroneocystostomy can be performed using a JJ catheter [2, 8]. Since normally the risk of strangulation is low, conservative management and follow-up can be chosen in cases with absent obstructive uropathy [5].

Regarding inguinal herniorrhaphy with mesh, there are no significant differences in terms of laparoscopic versus open surgery, with surgical experience and access to technology being the only variables considered to pick one procedure over the other $[1,9]$. Concerning UISH,

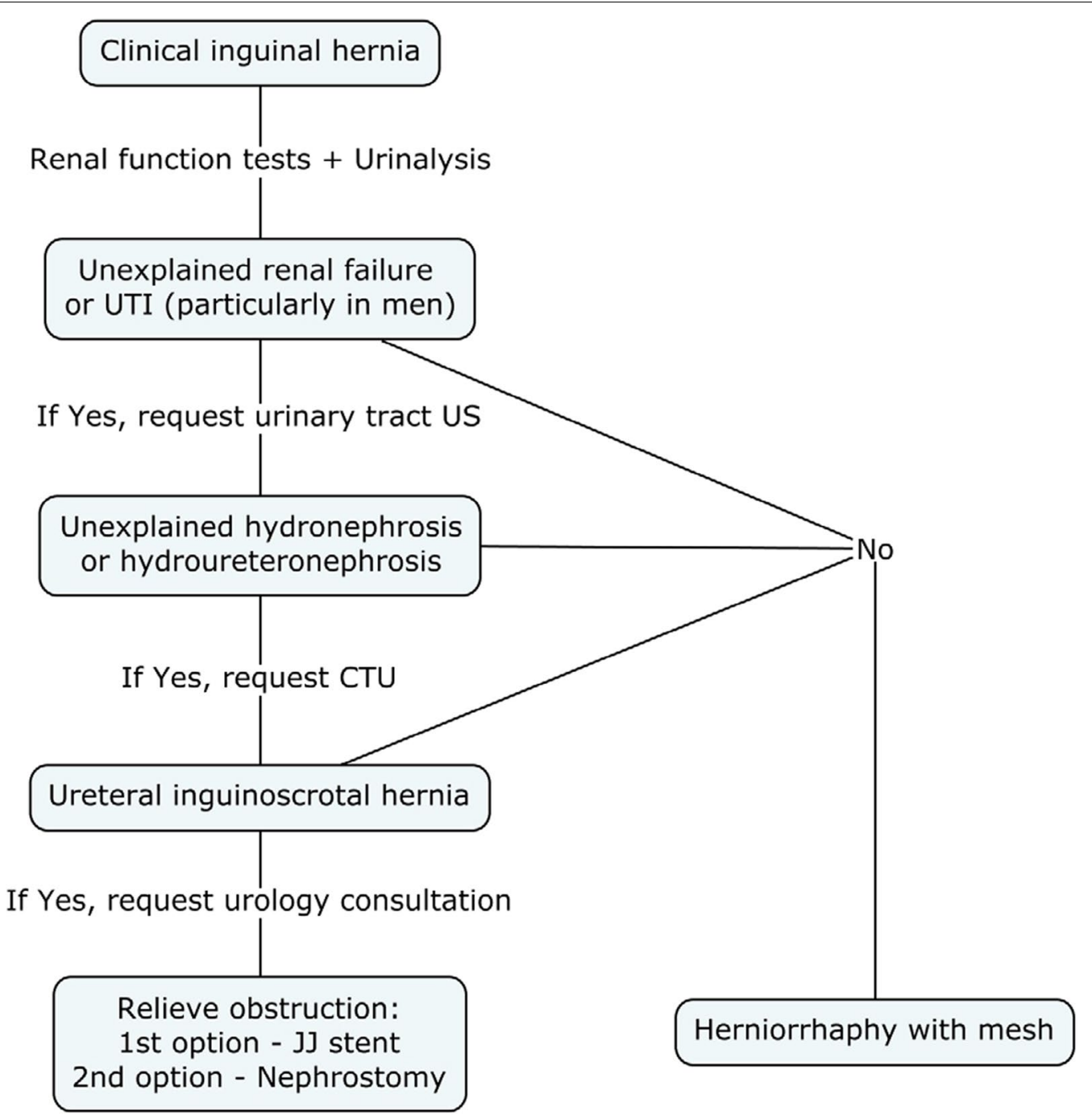

Fig. 6 Algorithm for ruling out and diagnosing ureteral inguinoscrotal hernia, adapted from Yahya et al. [1]. UT/ urinary tract infection, US ultrasound, CTU computed tomography urogram 
the experience of an open approach herniorrhaphy with mesh and two cases of robotic-assisted surgery have been reported $[16,17]$.

To our knowledge at the time of the review of the literature for the preparation of the article, this is the first reported case of a ureteral paraperitoneal inguinoscrotal hernia in a patient with a pelvic ectopic solitary kidney and malrotation. Currently, it is also the only case reported that has been resolved by laparoscopy.

\section{Conclusion}

The case here presented outlines the experience of managing a rare disease with a presentation that has not been described yet. Given the low prevalence of this pathology, case series, reports, and experiences of medical centers are important to improve its management and outcomes.

\begin{abstract}
Abbreviations
UISH: Ureteral inguinoscrotal hernia; NIHSS: National Institutes of Health Stroke Scale; ASPECTS: Alberta Stroke Program Early CT Score; eGFR: estimated glomerular filtration rate; CKD-EPI: chronic kidney disease epidemiology collaboration; US: ultrasound; CTU: computed tomography urogram; CT: computed tomography; UTI: urinary tract infection.
\end{abstract}

\section{Acknowledgements}

None.

\section{Authors' contributions}

JCA was involved in conception, design, supervision, materials and data collection and processing, analysis and interpretation, literature review, writing, and critical review. SJMD and SGC had contributed to data collection and processing, analysis and interpretation, and literature review. CAR participated in writing and critical review. DSR and JBC took part in supervision and critical review. All authors read and approved the final manuscript.

\section{Funding}

There is no funding for this report.

\section{Availability of data and materials}

Upon acceptance, the authors agree to the copyright rules of the African Journal of Urology Case Reports.

\section{Declarations}

\section{Ethics approval and consent to participate}

Written consent was obtained from the patient to publish the case report.

\section{Consent for publication}

Written consent for publication was obtained from the patient.

\section{Competing interests}

The authors declare that they have no competing interests.

\section{Author details}

${ }^{1}$ Department of Urology, School of Medicine, Universidad Nacional de Colombia, Carrera 30 No. 45-03, Edificio 471, Bogotá, Colombia. ${ }^{2}$ Department of Surgery, Fundación Universitaria Sanitas, Bogotá, Colombia. ${ }^{3}$ School of Medicine, Universidad del Norte, Barranquilla, Colombia. ${ }^{4}$ School of Medicine, Universidad Nacional de Colombia, Bogotá, Colombia. ${ }^{5}$ Department of Urology, School of Medicine, Universidad Militar Nueva Granada, Bogotá, Colombia. ${ }^{6}$ Department of Surgery, School of Medicine, Fundación Universitaria San Martín, Bogotá, Colombia.

Received: 17 January 2021 Accepted: 15 April 2021

Published online: 28 April 2021

\section{References}

1. Yahya Z, Al-Habbal Y, Hassen S. Ureteral inguinal hernia: an uncommon trap for general surgeons. BMJ Case Rep. 2017;2017.

2. Malde S, Bilagi P, Marsh H (2013) Obstructive uropathy due to ureteroinguinal hernia: an uncommon occurrence. Indian J Urol 29(4):355-356

3. Sidiqi MM, Menezes G (2018) Asymptomatic herniation of ureter in the routine inguinal hernia: a dangerous trap for general surgeons. Int J Surg Case Rep 49:244-246

4. Zarraonandia Andraca A, Ríos Reboledo Á, Casas Nebra J, Ponce DíazReixa J, Martínez Breijo S, González Dacal J et al (2009) Hernia ureteral inguinal: caso clínico [Ureteroinguinal hernia: Clinical case]. Arch Esp Urol 62:755-757 (in Spanish)

5. Lu A, Burstein J (2012) Paraperitoneal inguinal hernia of ureter. J Radiol Case Rep 6(8):22-26

6. Winston J, Salinas J, Nuwayhid F (2020) Not just a simple inguinal hernia. A rare case of an inguino-scrotal ureter! Urol Case Rep. 28:101036

7. de Carvalho NR, Westin CEG, de Arruda JGF, de Arruda PFF, Gatti M, Bogdan AP et al (2018) Inguinal hernia with ureter in patient with single kidney. AME Case Rep 2:5

8. Dikmen AV, Guneri C, Yalcin S, Acikgoz O, Ak E, Cetiner S (2017) A ureteral inguinoscrotal hernia from a pelvic kidney. Curr Urol 11(1):51-53

9. Group H (2018) International guidelines for groin hernia management. Hernia 22(1):1-165

10. Öberg S, Andresen K, Rosenberg J (2017) Etiology of inguinal hernias: a comprehensive review. Front Surg 4:52

11. Pareja-López Á, Sevilla-Cecilia C, Pey-Camps A, Dominguez-Tristancho $J$, Muteb M (2015) Ureteroinguinal hernia. Cir Esp 93(8):e89-e90 (in Spanish)

12. Singhal U, Morhardt D, Davenport MS, Moussa MH, Alam H, Malaeb B et al (2016) Ureteral involvement within an incarcerated inguinal hernia in a patient with crossed-fused renal ectopia. Urol Case Rep 7(July):20-22

13. Sripathi S, Rajagopal K, Kakkar C, Polnaya A (2011) Case Report-Inguinoscrotal ureteral hernia diagnosed on micturating cystourethrography. Indian J Radiol Imaging 21(3):199-201

14. Auld MC, Gallagher K, Ali A (2019) Scrotal extraperitoneal ureteroinguinal hernia with a horseshoe kidney. ANZ J Surg 89(1-2):E39-E40

15. Allam ES, Johnson DY, Grewal SG, Johnson FE (2015) Inguinoscrotal herniation of the ureter: description of five cases. Int I Surg Case Rep. 14:160-163

16. Pucheril D, Chun B, Dalela D, Abdollah F, Laker SA, Rogers CG (2017) Robot-assisted laparoscopic repair of extraperitoneal ureteral inguinal hernia with mesh placement. J Endourol Case Rep 3(1):97-100

17. Cetrulo LN, Harmon J, Ortiz J, Canter D, Joshi AR (2015) Case report of a robotic-assisted laparoscopic repair of a giant incarcerated recurrent inguinal hernia containing bladder and ureters. Int J Med Robot 11(1):15-17 